\title{
Synthesis of Silver Nano Particles Using Herbal Extract and its Anti-Microbial Activity
}

\section{Bhoominathan Srinivasan*}

Department of biotechnology, Bharathidasan University, Tiruchurappali, India

\begin{abstract}
Microorganisms assume a critical part in lethal metal remediation through diminishment of metal particles. Studies showed that silver particles may be diminished additional cellularly utilizing Fusarium oxysporum to produce stable gold or silver nanoparticles in water. These particles can be joined in a few sorts of materials, for example, fabrics. These fabrics with silver nanoparticles are sterile and can be valuable in doctor's facilities to anticipate or to minimize disease with pathogenic microscopic organisms, for example, Staphylococcus aureus. In this work, the extracellular creation of silver nanoparticles by $F$. oxysporum and its antimicrobial impact when joined in cotton fabrics against $S$. aureus were examined.
\end{abstract}

Keywords: Nano materials; Nano particles; Microorganisms; Fusarium oxysporum

\section{Introduction}

Microorganisms play an important role in toxic metal remediation through reduction of metal ions. Studies demonstrated that silver ions may be reduced extra cellularly using Fusarium oxysporum to generate stable gold or silver nanoparticles in water. These particles can be incorporated in several kinds of materials such as cloths. These cloths with silver nanoparticles are sterile and can be useful in hospitals to prevent or to minimize infection with pathogenic bacteria such as Staphylococcus aureus. In this work, the extracellular production of silver nanoparticles by $F$. oxysporum and its antimicrobial effect when incorporated in cotton fabrics against $S$. aureus were studied. In addition, all effluent was bioremediated using treatment with $C$. violaceum. The results showed that cotton fabrics incorporated with silver nanoparticles displayed a significant antibacterial activity against $S$. aureus. The effluent derived from the process was treated with $C$. violaceum and exhibited an efficient reduction in the silver nanoparticles concentration. In conclusion, it was demonstrated the application of biological synthesis to silver nanoparticles production and its incorporation in cloths, providing them sterile properties. Moreover, to avoid any damage to the environment the effluent containing silver nanoparticles can be treated with cyanogenic bacterial strains [1].

Water is one of the essential enablers of life on earth. Beginning with the origin of the earliest form of life in seawater, it has been central to the evolution of human civilizations. Noble metals have been similarly associated with the prosperity of human civilizations through their prominent use in jewellery and medical applications. The most important reason for the use of noble metals is the minimal reactivity at the bulk scale, which can be explained by a number of concepts such as electrochemical potential, relativistic contraction, molecular orbital theory, etc. Recently, water quality has been associated with the development index of society. A number of chemical and biological contaminants have endangered the quality of drinking water. An overview of important events during last 200 years in the area of drinking water purification is presented. Realizing the molecular nature of contamination in drinking water, significant progress has been made to utilize the chemistry of nano materials for water purification. This article summarizes recent efforts in the area of noble metals nano particle synthesis and the origin of their reactivity at the nanoscale. The application of noble metal nanoparticle based chemistry for drinking water purification is summarized for three major types of contaminants: halogenated organics including pesticides, heavy metals and microorganisms. Recent efforts for the removal, as well as ultra-low concentration detection of such species, using noble metal nano particles are summarized. Important challenges during the commercialization of nano based products are highlighted through a case study of pesticide removal using noble metal nano particles. Recent efforts in drinking water purification using other forms of nano materials are also summarized. The article concludes switch recent investigations on the issue of nano toxicity and its implications for the future [2].

Curcumin, a yellow polyphenol extracted from the rhizome of turmeric (Curcuma longa), has potent anti-cancer properties as demonstrated in a plethora of human cancer cell line and animal carcinogenesis models. Nevertheless, widespread clinical application of this relatively efficacious agent in cancer and other diseases has been limited due to poor aqueous solubility, and consequently, minimal systemic bioavailability. Nanoparticle based drug delivery approaches have the potential for rendering hydrophobic agents like curcumin dispersible in aqueous media, thus circumventing the pitfalls of poor solubility.

In this paper the synthesis of silver nanoparticles using Parthenium leaf extract is described. Transmission electron microscopy analysis of these particles shows that they are $\sim 50 \mathrm{~nm}$ in range and assembled in very irregular shape of variable morphology. The most needed outcome of this work will be the development of value-added products from Parthenium for biomedical and nanotechnology based industries [3].

Developing bactericidal coatings using simple green chemical methods could be a promising route to potential environmentally friendly applications. Here, we describe an environmentally friendly chemistry approach to synthesize metal-nanoparticle (MNP)embedded paint, in a single step, from common household paint.

*Corresponding author: Bhoominathan Srinivasan, Department of biotechnology, Bharathidasan University, Tiruchurappali, India, Tel: 9633946831; E-mail: srinivas9876@gmail.com

Received February 14, 2015; Accepted February 24, 2015; Published February 04, 2015

Citation: Srinivasan B (2015) Synthesis of Silver Nano Particles Using Herbal Extract and its Anti-Microbial Activity. J Phylogen Evolution Biol 3: 146. doi:10.4172/2329-9002.1000146

Copyright: (c) 2015 Srinivasan B. This is an open-access article distributed under the terms of the Creative Commons Attribution License, which permits unrestricted use, distribution, and reproduction in any medium, provided the original author and source are credited. 
The naturally occurring oxidative drying process in oils, involving free-radical exchange, was used as the fundamental mechanism for reducing metal salt sand dispersing MNPs in the oil media,without the use of any external reducing or stabilizing agents. These well-dispersed MNP-in-oil dispersions can be used directly, akin to commercially available paints, on nearly all kinds of surface such as wood, glass, steel and divergent polymers. The surfaces coated with silver-nanoparticle paint showed excellent antimicrobial properties by killing both Grampositive human pathogens (Staphylococcus aureus) and Gram-negative bacteria (Escherichia coli). The process we have developed here is quite general and can be applied in the synthesis of a variety of MNP-in-oil systems.

The antimicrobial effects of silver (Ag) ion or salts are well known, but the effects of Ag nanoparticles on microorganisms and antimicrobial mechanism have not been revealed clearly. Stable Ag nanoparticles were prepared and their shape and size distribution characterized by particle characterizer and transmission electron microscopic study. The antimicrobial activity of Ag nano particles was investigated against yeast, Escherichia coli, and Staphylococcus aureus. In these tests, Muller Hinton agar plates were used and Ag nano particles of various concentrations were supplemented in liquid systems. As results, yeast and E. coli were inhibited at the low concentration of $\mathrm{Ag}$ nano particles, whereas the growth-inhibitory effects on $\mathrm{S}$. aureus were mild. The freeradical generation effect of $\mathrm{Ag}$ nanoparticles on microbial growth inhibition was investigated by electron spin resonance spectroscopy. These results suggest that $\mathrm{Ag}$ nano particles can be used as effective growth inhibitors in various microorganisms, making them applicable to diverse medical devices and antimicrobial control systems.

Bactericidal activity of a malodor neutralizer containing silver nanoparticles manufactured in Iran has been tested. For this purpose different concentration of the product encounter with Staphylococcus aureus, Pseudomonas aeroginosa and Bacillus cereus then the activity were evaluated in different contact times. The product which contains at least $200 \mathrm{ppm}$ of silver nano particles was effective on all of bacterial strain and increase of contact time lead to improvement in this effect. Bactericidal activity against spore of Bacillus cereus is less in comparison with other bacteria. It seems that silver nanoparticles have a good antibacterial activity even in presence of aromatic fragments. Therefore could use as disinfectant in many situation.

The antibacterial properties of differently shaped silver nanoparticles against the gram-negative bacterium Escherichia coli, both in liquid systems and on agar plates. Energy-filtering transmission electron microscopy images revealed considerable changes in the cell membranes upon treatment, resulting in cell death. Truncated triangular silver nanoplates with a $\{111\}$ lattice plane as the basal plane displayed the strongest biocidal action, compared with spherical and rod-shaped nanoparticles and with $\mathrm{Ag}+$ (in the form of $\mathrm{AgNO}_{3}$ ). It is proposed that nanoscale size and the presence of a $\{111\}$ plane combine to promote this biocidal property. To our knowledge, this is the first comparative study on the bactericidal properties of silver nanoparticles of different shapes, and our results demonstrate that silver nanoparticles undergo a shape-dependent interaction with the gram-negative organism E. coli.

An optimal set of conditions for the synthesis of clear yellow colloidal silver was investigated. Silver nanoparticles were obtained by borohydride reduction of silver nitrate, a method which produces particles with average size of $12 \pm 2 \mathrm{~nm}$, determined by Scanning Electron Microscopy (SEM). The Plasmon absorbance is at $397 \mathrm{~nm}$ and the
Peak Width at Half Maximum (PWHM) is 70-75 nm. The relationship between aggregation and optical properties was determined along with a method to protect the particles using polyvinyl pyrrolidone (PVP). A laboratory experiment was designed in which students synthesize yellow colloidal silver, estimate particle size using visible spectroscopy, and study aggregation effects.

Nanotechnology can be applied in the production, processing, safety and packaging of food. It is possible that nanotechnology will allow the manipulation of the molecular forms of food to provide more capability, lower costs and greater sustainability than at present. A nanocomposite coating process should improve food packaging by placing anti-microbial agents directly on the surface of the coated film and could increase or decrease gas permeability as required for different products. They can also improve the mechanical and heatresistance properties and lower the oxygen transmission rate. It should also be possible to apply nanotechnology to the detection of chemical and biological substances for sensing biochemical changes in foods, extending to the whole food chain in the future.

As reviewed by Salata, nanomaterials are also being used in biology and medicine in a wide variety of ways, including the direct application of products into patients. Examples include products for drug delivery and gene therapy, the separation and purification of biological molecules and cells, fluorescent biological labels, imaging contrast agents, tissue engineering, DNA probes and nanoscale biochips, and microsurgical technology.

Nanomaterials are, of course, abundant in nature as living organisms operate basically at a nanoscale level. Nanotechnologists seek to produce and utilize both novel nanomaterials and some natural nanomaterials in larger quantities and within a more consistent size range. Numerous techniques are used to fabricate different nanomaterials. Nanoparticles can be produced grinders, lasers, and vaporization followed by cooling. For complex particles, nanotechnologists generally prefer to synthesize nanostructures by a bottom-up approach by arranging molecules to form complex structures with new and useful properties.

Nanomaterials possess various new properties and their industrial use creates new opportunities, but they also present new risks and uncertainties. Growing production and use of nanomaterials result in an increasing number of workers and consumers exposed to nanomaterials. This leads to a greater need for information on possible health and environmental effects of nanomaterials. (The European Agency for Safety and Health at Work released a literature review entitled "Workplace exposure to nanoparticles").

It's the revolutionary science and art of manipulating matter at the atomic or molecular scale. The objective of nanotechnology is to produce materials of special function with new physical and chemical properties by making atoms and molecules behave differently. Nanotechnology is considered to be one of the most important sites of investment funded by the governorates.

\section{Materials and Methods}

\section{Parthenium leaf extract}

Principle: The use of environmentally benign materials like plant extract for synthesis of silver nanoparticles offers numerous benefits of ecofriendly and compatibility for pharmaceutical and biomedical applications. The synthesis of silver nanoparticles, reducing silver ions present in the aqueous solution of silver nitrate complex by the extract of Parthenium hysterophorus leaves, can provide a new platform to this 
noxious plant making it a value added weed for nanotechnology based industries in future.

Methodology: Extract has been prepared by bringing fresh leaves of Parthenium hysterophorus. Leaves weighing $25 \mathrm{~g}$ were thoroughly washed thrice in distilled water for $15 \mathrm{~min}$, cut into fine pieces and were boiled in a $500 \mathrm{ml}$ Erlenmeyer flask with $100 \mathrm{ml}$ distilled water up to 5 min and were filtered. Add $50 \mathrm{ml}$ leaf extract into the aqueous solution of $1 \mathrm{mM}$ Silver nitrate. An average size of the particles synthesized was $50 \mathrm{~nm}$ with size range 30 to $80 \mathrm{~nm}$ with irregular shape. Due to our interest to get much smaller particles, above solution was centrifuged at a rate of $1200 \mathrm{rpm}$ up to $15 \mathrm{~min}$ and investigated that particles present in the supernatant were nearly homogenous with average size of $7 \mathrm{~nm}$.

The reduction of pure Ag ions was monitored by measuring the UV-Vis spectrum of the reaction medium at different time intervals after diluting a small aliquot of 100 micro litres of the sample with 1 $\mathrm{ml}$ deionized water. UV-vis spectral analysis has been done by using a Perkin- Elmerlamda-25 spectrophotometer.

The reaction mixture was kept for 7 days at room temperature for stabilization and subsequently it was centrifuged at $8000 \mathrm{rpm}$ for 5 minutes and redispersed in distilled water. This procedure was repeated three times and the remnant pellets were dried and powdered for SEM analysis. A thin film of the sample was prepared by dissolving a portion of the powdered particles in sterile distilled water on a small glass cover slip $(3 \times 3 \mathrm{~mm})$, and set on a copper stab for electron microscopy. Analysis was done using Scanning Electron Microscope (SEM) at IICT, Hyderabad.

\section{Antibacterial activity of silver nano particles - by disc method}

For the anti-microbial activity, different bacteria like E. coli, Pseudomonas, Salmonella, Staphylococcus and fungi like Aspergillus and Pencillum were obtained from IMTECH, Chandigrah, INDIA. These cultures were sub-cultured in the nutrient broth for further use.

Antimicrobial activity of silver nano particles synthesised using Parthenium extract was measured by Kirby Bauer method against bacteria like E. coli, Pseudomonas, Salmonella, Staphylococcus and fungi like Aspergillus and Pencillum. Nutrient Agar plates with specific cultures were incubated for $24 \mathrm{hrs}$.

The filter paper discs which were coated with silver nano particles $50 \mathrm{mg} / \mathrm{lt}$ were placed on to the surface of agar plates. The zone of inhibition after $24 \mathrm{hrs}$ of incubation at $37^{\circ} \mathrm{C}$ was recorded. The disc without nano particle was used as negative control.

\section{Incorporating silver nanoparticles on cotton fabric}

The cotton cloth was immersed in the nanoparticle solution synthesized using Parthenium extract. This was centrifuged (3500 rpm) for 15 minutes. Later it was dried and used to test for antimicrobial activity by Kirby Bauer method.

\section{Results and Discussions}

\section{Synthesis of silver nanoparticle using Parthenium leaf extract}

The color change in the colloidal solution of nanoparticles reduced by Parthenium plant leaf extract with time (in the inset) is shown in Figure 1.

UV-Vis spectrograph of the colloidal solution of silver nanoparticles has been recorded as a function of time. Absorption spectra of silver nanoparticles formed in the reaction media at $10 \mathrm{~min}$ has absorbance peak at $474 \mathrm{~nm}$, broadening of peak indicated that the particles are poly dispersed Figure 2.

\section{SEM analysis of silver nano particles}

SEM Micrograph (Figure 3) of the silver nanoparticles synthesized using Parthenium leaf extract having irregular shapes of 30 to $80 \mathrm{~nm}$ with average size $50 \mathrm{~nm}$.

Reduction of silver ions present in the aqueous solution of silver complex during the reaction with the ingredients present in the plant leaf extract observed by the UV-Vis spectroscopy revealed that silver nanoparticles in the solution may be correlated with the UV-Vis spectra. As the Parthenium leaf extract was mixed in the aqueous solution of the silver ion complex, it started to change color from water color to yellowish brown (Figure 4), color was changed due to excitation of surface plasmon vibrations, which indicated formation of silver nanoparticle. UV-Vis spectroscopy is well known to investigate shape and size controlled of nanoparticles. UV-Vis spectrograph of the colloidal solution of silver nanoparticles has been recorded as a function of time by using a quartz cuvette with water as reference, repeated experiments were carried out with varying the amount of silver ion complex $(1 \mathrm{mM})$ and leaf extract it was observed that precursors in the ratio of 1:1 gave best results of our interest. It is interesting to note that most of the particles in the SEM pictures are not in physical contact but are separated by a fairly uniform inter particle distance.

Not only the physicists and chemists, but also the biologists are highly interested in synthesizing nanoparticles of different shapes and sizes by employing bio-based synthesis of nanometals using plant leaf extracts and microorganisms (fungi and bacteria). The reduction of silver ions $\left(\mathrm{Ag}^{+}\right)$present in the aqueous solution of silver complex in the plant extract demonstrated that the change in colour was due to the formation of silver nanoparticles in the solutions which are correlated with the UV-Vis spectra [3]

The eco-friendly green chemistry approach for the use of these weeds for synthesis of silver nanoparticles will increase their economic viability and sustainable management. However, applications of these weeds have the added advantage that these unwanted plants can be used by nanotechnology processing industries as well in bactericidal, wound healing and other medical and electronic applications [4].

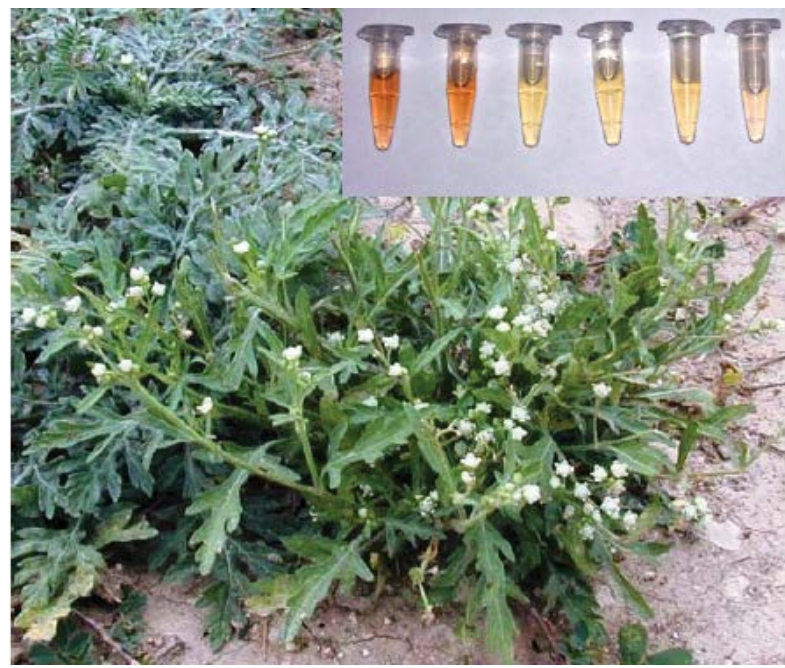

Figure 1: Parthenium plant and in the inset optical photograph of the colloidal solution of silver nanoparticles reduced by Parthenium leaf extract 
Citation: Srinivasan B (2015) Synthesis of Silver Nano Particles Using Herbal Extract and its Anti-Microbial Activity. J Phylogen Evolution Biol 3: 146. doi:10.4172/2329-9002.1000146

Page 4 of 5

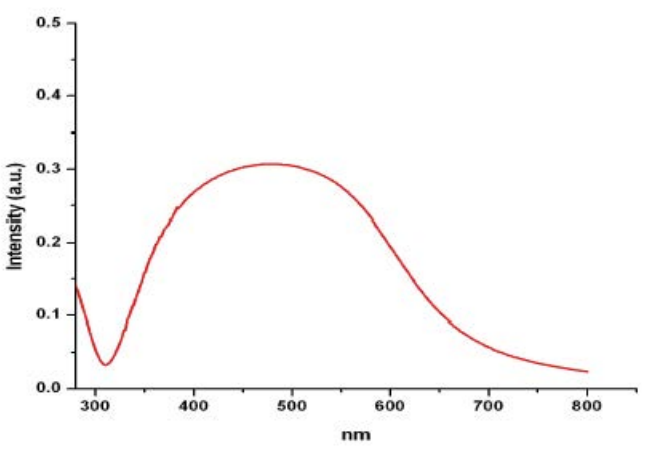

Figure 2: UV-Vis. Absorption spectra recorded as a function of time of reaction of $1: 1$ solution of silver ions by Parthenium leaf extract in the range $300 \mathrm{~nm}$ to $800 \mathrm{~nm}$ after 10 minutes reaction kinetics.

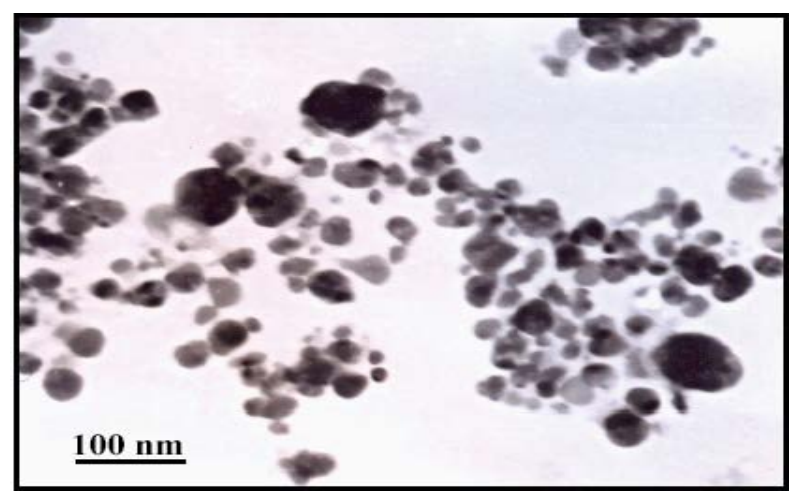

Figure 3: SEM Micrograph of the sample after the 10 minute reaction kinetics with treating leaf extract with silver ions complex $(1 \mathrm{mM})$ in the ratio of $1: 1$, showing particles of irregular shapes which varies in size from $30 \mathrm{~nm}$ to $80 \mathrm{~nm}$ (average particle size is $50 \mathrm{~nm}$ ).

\section{Antimicrobial properties of nanoparticles}

Antimicrobial activity of silver nano particle from Parthenium extract was measured by Kirby Bauer method against bacteria like E. coli, Pseudomonas, Salmonella, Staphylococcus and fungi like Aspergillus and Pencillum. Nutrient Agar plates with specific cultures were incubated $24 \mathrm{hrs}$. The filter paper discs which were coated with silver nano particles $50 \mathrm{mg} /$ lit were placed on to the surface of agar plates. The zone of inhibition after $24 \mathrm{hrs}$ incubation at $37^{\circ} \mathrm{C}$ was recorded. The disc without nano particle was used as negative control.

Our result indicated that silver nano particles synthesized from herbal extract were more efficient against bacteria compared to fungi (Figures 4-9). The Nano particles show the antibacterial activity against both gram positive and gram negative bacteria. Comparing the zone of inhibitions, it can be concluded that the silver nano particles have greatest antibacterial against Salmonella and least against $E$. coli. However there is not much of a difference in antifungal activity between the two fungal species (Table 1).

\section{Antimicrobial properties of nanoparticles incorporated in cloth}

The antibacterial activity of cotton fabrics with and without silver nanoparticles was evaluated. Since the nano particles in the previous experiment did not show good response against fungal $\mathrm{sp}$, the cotton cloth was analyzed only against bacterial cultures. In the cloth without silver nanoparticles (control) a significant bacterial growth was observed.

The cotton fabrics with silver nano particles synthesised using Parthenium extract exhibited antibacterial activity as shown in Figures 10a-d). Comparing the zone of inhibitions it can be concluded that the silver nano particles have greatest antibacterial against Salmonella and least against Staphlococcus and Pseudomonas (Table 2).

The cotton fabrics incorporated with these silver nanoparticles exhibited maximum antibacterial activity against Salmonella Sp. This study demonstrated the possibility of using silver nanoparticles by incorporating them in fabrics, thereby providing them sterile properties.

\begin{tabular}{|c|c|}
\hline Microorganism & Zone of inhibition(cm) \\
\hline Salmonella Sp & 1.2 \\
\hline Staphylococcus Sp & 0.8 \\
\hline Pseudomonas Sp & 0.7 \\
\hline E. coli Sp & 0.5 \\
\hline Aspergillus Sp & 0.2 \\
\hline Pencillium Sp & 0.3 \\
\hline
\end{tabular}

Table 1: Comparing the zone of inhibitions formed by nano particles

\begin{tabular}{|c|c|}
\hline Microorganism & Zone of inhibition (cm) \\
\hline Salmonella $S p$ & 0.6 \\
\hline Staphylococcus $S p$ & 0.2 \\
\hline Pseudomonas $S p$ & 0.2 \\
\hline E. coli $S p$ & 0.5 \\
\hline
\end{tabular}

Table 2: Measure of antimicrobial activity on different bacterial species

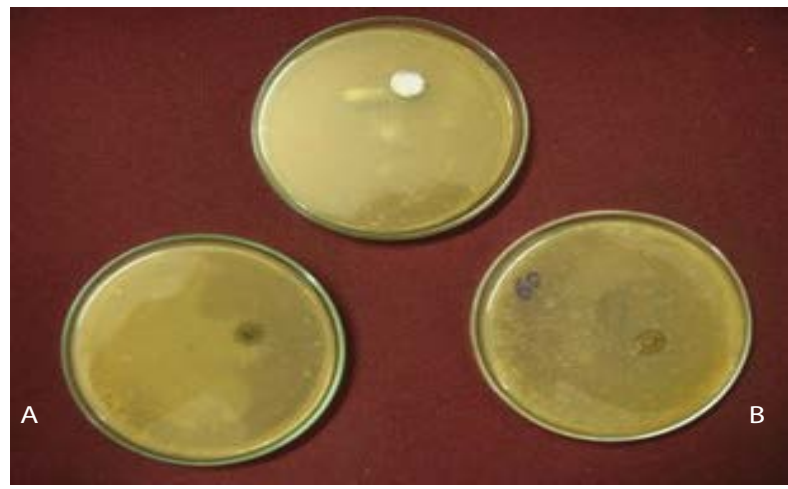

Figure 4: Antimicrobial activity against Salmonella Sp.

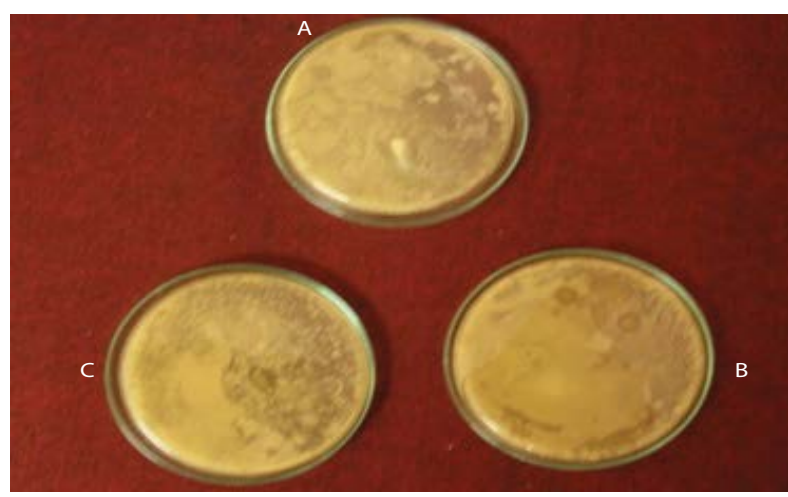

Figure 5: Antimicrobial activity against Staphylococcus $S p$. 
Citation: Srinivasan B (2015) Synthesis of Silver Nano Particles Using Herbal Extract and its Anti-Microbial Activity. J Phylogen Evolution Biol 3: 146. doi:10.4172/2329-9002.1000146

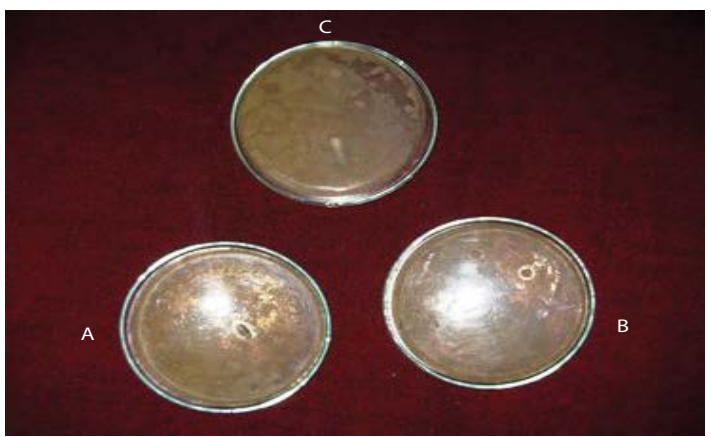

Figure 6: Antimicrobial activity against Pseudomonas Sp.

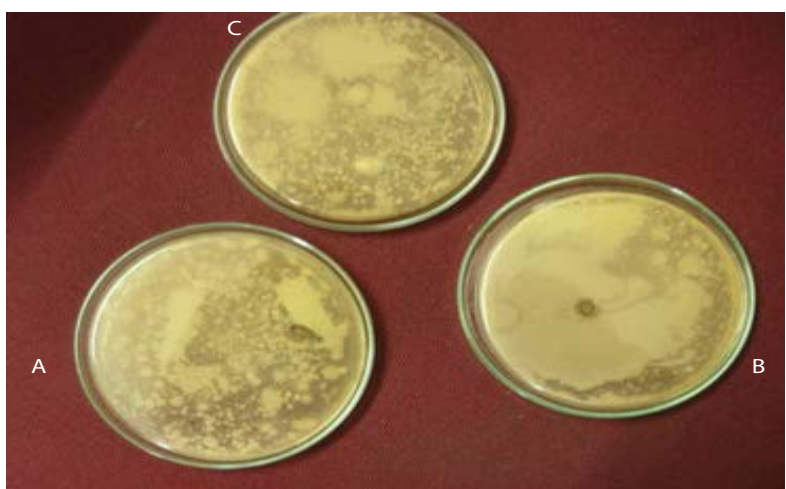

Figure 7: Antimicrobial activity against E. coli Sp.

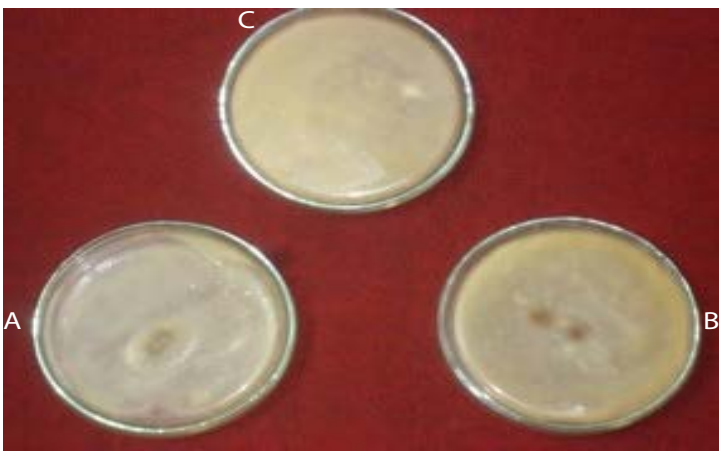

Figure 8: Antimicrobial activity against Aspergillus Sp.

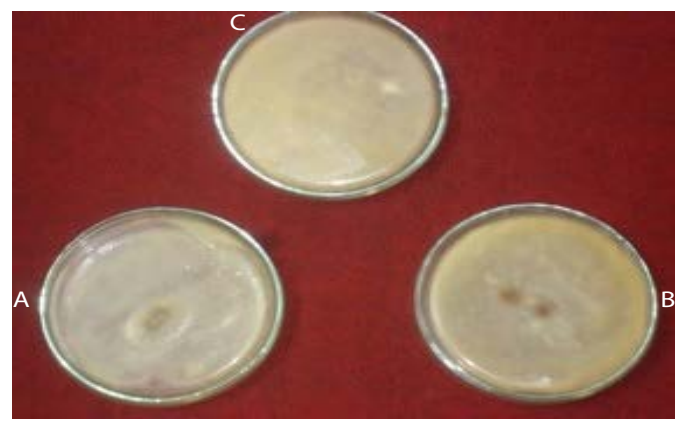

A-nano particles from chemical synthesis

B-nano particles from Parthenium extract

C-control

Figure 9: Antimicrobial activity against Pencillium Sp.

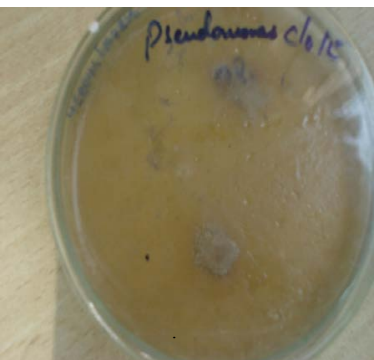

(a) Pseudomonas $S p$

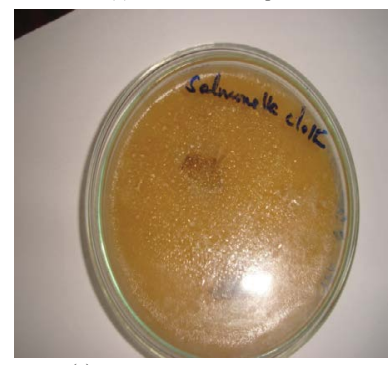

(c) Salmonella Sp.

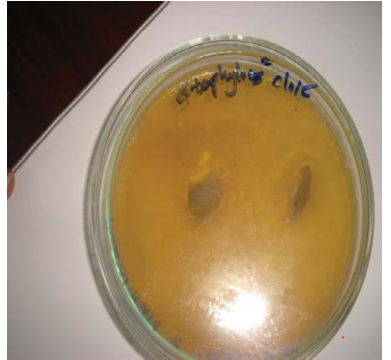

(b) Staphylococcus $S_{p}$

(d) E.coli $S p$

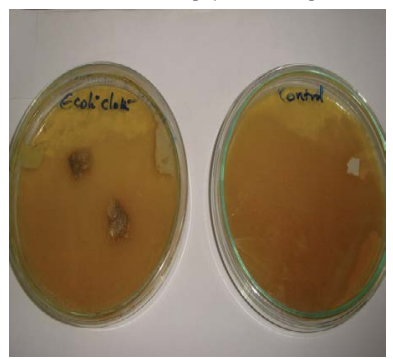

Figure 10: Antibacterial activity of cotton fabric coated with nanoparticles synthesized using Parthenium extract

\section{References}

1. Nelson Durán, Marcato PD, De Souza GIH, Alves OL, Esposito E (2005) Antibacterial Activity of Silver Nanoparticles Synthesized by Fusarium.

2. Pradeep T, Anshup (2009) Thin Solid Films. 517: 6441-6478

3. Parashar V, Parashar R, Sharma B, Pandey AC (2009) "Parthenium leaf extract mediated synthesis of silver nanoparticles: a novel approach towards weed utilization". Digest $\mathrm{J}$ of Nanomaterials and Biostructures 4: 45-50

4. Roy N, Barik A (2010) Green Synthesis of Silver Nanoparticles from theUnexploited Weed Resources. International Journal of Nanotechnology and Applications 4: 95-101 